\section{CEREBRO-SPLANCHNIC OXYGENATION IN EXTREMLY PRETERM INFANTS AND ITS CORRELATION WITH ENTERAL FEEDING}

E. Deschmann, M. Vanpee, M. Bartocci

Neonatal Unit, Karolinska Institutet, Stockholm, Sweden

Background: Early feeding of extremely preterm (EP) infants is essential for the development of the gastrointestinal tract. Nevertheless, optimal feeding strategy is unclear. Near infrared spectroscopy (NIRS), measuring cerebro-splanchnic oxygenation ratio (CSOR), detects in neonates changes in splanchnic oxygen delivery during apnea and predicts ischemia.

Aim: To study variations of CSOR in EP infants in relationship to initiation of enteral feeds.

Design/methods: In 9 EP infants born < 28 weeks gestational age, NIRS measurements were recorded: before (T0), directly after (T1), one day (T2) and one week after (T3) initiation of feeds. Clinical variables were recorded including the primary outcome: time to full feeds.

Results: Patients characteristics and outcomes in mean \pm SD or number of patients were: gestational age: $25.2 \pm 0.9$ weeks, birth weight: $764 \pm 134$ grams, sex: 4 females, Apgar score at 10 minutes: $8 \pm 2.06$, time to full feeds: $19 \pm 7$ days, incidence of necrotizing enterocolitis: 2, patent ductus arteriosus (PDA) treated with ibuprofen: 2, PDA ligation: 6, mortality rate: 1.

We observed significant differences between each period for individual patients. Mean CSOR values for each period were $1.4 \pm 0.4,1.7 \pm 0.9,1.5 \pm 0.7$, and $1.6 \pm 0.9$ for $\mathrm{T} 0, \mathrm{~T} 1, \mathrm{~T} 2$ and $\mathrm{T} 3$ respectively (NS). We found a correlation between CSOR at T3 and percent of enteral feeds $(p=0.0533, r=0.489)$, adjusted for birth weight $(p=0.0492, r=0.501)$. Six infants requiring PDA ligation showed higher CSOR values at T3: $1.908 \pm 0.92$ compared to $0.913 \pm 0.116$ (NS).

Conclusion: This pilot study suggests that CSOR can be used in EP infants to assess feeding intolerance and to optimize individual feeding strategies.

\section{REPRODUCIBILITY OF CEREBRAL TISSUE OXYGEN SATURATION MEASUREMENTS BY NEAR INFRARED SPECTROSCOPY IN NEWBORN INFANTS}

\author{
C. Jenny, M. Biallas, I. Trajkovic, M. Wolf
}

Clinic of Neonatology, University Hospital Zurich, Zurich, Switzerland

Objectives: Cerebral tissue oxygen saturation (StO2) may be a relevant parameter to assess brain oxygenation in intensive care. Near infrared spectroscopy (NIRS) is able to measure this parameter, but so far the reproducibility of such measurements has been too low due to inhomogeneities. The aim of this study was to test a novel sensor geometry, which is based on two source and two detector locations, which are arranged symmetrically to reduce the influence of superficial inhomogeneities.

Methods: Thirty clinically stable, spontaneously breathing newborn infants with a gestational age of $34.2 \pm 3.1$ weeks, birth weight of $2295 \pm 970 \mathrm{~g}$ and postnatal age of $10.2 \pm 14.9$ days were measured four times for one minute by NIRS on the lateral head. Between measurements the sensor was replaced. The reproducibility was calculated by a linear mixed effects model.

Results: The mean StO2 was $79.99 \pm 4.47 \%$ with a reproducibility of $2.76 \%$ and a variability of $4.20 \%$ between infants. The error of measurement only accounts for $30 \%$ of the variability.

Conclusions: The novel sensor geometry leads to much more precise measurements compared to previous studies (e.g. $\sim 5 \%$ reproducibility for the $\mathrm{NIRO} 300$ ). For this reason the measured StO2 values do not require averaging and are therefore more clinically relevant.

Acknowledgments: The authors gratefully acknowledge funding of the Swiss National Science Foundation. 